'Departamento de Educación Médica Facultad de Medicina, Universidad de Concepción. Concepción, Chile. ${ }^{2}$ Facultad de Medicina, Universidad Andrés Bello. Viña del Mar, Chile.

${ }^{3}$ Departamento Obstetricia y Ginecología, Oficina de Educación en Ciencias de la Salud (OFECS), Facultad de Medicina, Universidad de La Frontera. Temuco, Chile. ${ }^{4}$ Departamento de Kinesiología, Facultad de Medicina, Universidad de Concepción. Concepción, Chile.

angeniero Informático, Magíster en Educación Médica para las Ciencias de la Salud.

bsicóloga, Magíster en Ciencias de la Educación, mención Didáctica e Innovación Pedagógica

'Psicólogo, Doctor en Ciencias de la Educación

dMatrona, Doctora en Desarrollo Profesional e Institucional para la calidad educativa. ePsicólogo, Magíster en Bioestadística. ${ }^{f}$ Enfermera, Magíster en Envejecimiento y Calidad de Vida.

${ }^{9}$ Kinesióloga, Magíster en Salud Pública Mención Gerontología Social. hPsicóloga.

iPsicóloga, Magíster en Psicología Educacional. jpsicólogo

Fuente de apoyo Financiero: ConicityFondecyt Regular N 1170525

Título del proyecto: Diversidad e inclusión educativa en carreras de la salud en universidades chilenas.

Los autores declaran no tener conflictos de interés.

Recibido el 25 de julio de 2019, aceptado el 20 de marzo de 2020

Correspondencia a: Olga Matus-Betancourt Chacabuco esquina Janequeo S/N Concepción, Chile. omatus@udec.c

\section{Formación clínica en carreras de la salud: ¿ dónde se incluye la diversidad?}

\author{
OLGA MATUS-BETANCOURT 1,a, JAVIERA ORTEGA- \\ BASTIDAS $^{1, \mathrm{~b}}$, CRISTHIAN PÉREZ-VILLALOBOS ${ }^{1, \mathrm{c}}$, \\ PETER MCCOLL-CALVO ${ }^{2}$, NANCY NAVARRO-HERNÁNDEZ 3 , , \\ VERÓNICA SILVA-ORREGO ${ }^{2}$, JUAN ARELLANO-VEGA ${ }^{1, \mathrm{e}}$, \\ IVONE CAMPOS-CERDA ${ }^{1, \mathrm{f}}$, MARÍA JOSÉ SOLÍS-GRANT ${ }^{4, \mathrm{~g}}$, \\ MARY JANE SCHILLING-NORMAN ${ }^{1, \mathrm{~h}}$, CAMILA \\ ESPINOZA-PARCET ${ }^{1, \mathrm{i}}$, SEBASTIÁN GONZÁLEZ-BREVIS ${ }^{1, \mathrm{j}}$
}

\section{The inclusion of diversity in clinical training}

Background: Educational inclusion, a concept that has changed over time, is becoming relevant. It initially considered only disability and now contemplates education for all people. Educational inclusion has special relevance in health care workers' training, who will have direct contact with heterogeneous populations, where diversity-oriented treatments are required. Aim: To describe how diversity is integrated into health care training in a clinical context. Material and Methods: A qualitative study, based on Grounded Theory. Two group interviews and thirteen semi-structured interviews were conducted among 11 teachers and 25 students of health careers. Data was analyzed using Atlas-ti 7.5.2. A constant comparison method, reaching an axial coding level, was used. Results: The category of training in health careers in the context of diversity emerged. It is a process that operates in cognitive, affective and behavioral dimensions. It accounts for the diversity of system actors and the model of a health professional and teacher facing diversity. Conclusions: These dimensions should be understood during clinical training. Socio-cultural diversity encompassing gender, sex, ethnicity, religion and disability should be considered. Therefore, educational inclusion is an important issue to be considered by universities.

(Rev Med Chile 2020; 148: 444-451)

Key words: Clinical Clerkship; Cultural Diversity; Education, Medical.
$\mathrm{D}$ esde principios del siglo XXI, la discusión en educación médica ha enfatizado la necesidad de repensar el ejercicio pedagógico, recuperando principios de la andragogía que buscan promover el aprendizaje profundo y autónomo en los estudiantes ${ }^{1,2}$. Junto con ello, la formación es pensada desde un abordaje inte- gral, que permite a los estudiantes apropiarse del enfoque biopsicosocial en salud ${ }^{3,4}$. Esto requiere pensar sobre aquellas condiciones que fundan el proceso formativo en carreras de la salud ${ }^{5} y$ reconocer cómo el docente guía la experiencia formativa en el contexto clínico. En un estudio realizado en carreras de la salud chilenas fue po- 
sible evidenciar que los docentes suelen actuar de dos modos en el escenario clínico: 1) cumpliendo un rol docente-asistencial que se caracteriza por basar su enseñanza en la experiencia que han ido adquiriendo a lo largo de su experiencia como profesionales de la salud o 2) cumpliendo un rol de enseñanza espontánea, sujeta principalmente a las características dinámicas, aleatorias y complejas del contexto clínico ${ }^{6}$. Pero allende los roles docentes, falta aún comprender mejor cómo opera la formación clínica en carreras de la salud, no solo desde el punto de vista del docente y del estudiante, sino también desde el punto de vista del paciente. En esta formación, la diversidad adquiere relevancia por tres razones: 1) quienes actúan en este contexto son diversos; 2) formar un profesional sanitario requiere preparar para la diversidad y 3 ) la educación formal debe considerar los principios de la inclusión educativa.

La inclusión educativa se refiere al derecho de la persona a pertenecer y participar en el sistema educativo, suponiendo que este sistema reconozca las diferencias propias de los participantes que actúan en él ${ }^{7}$. En la actualidad, los sistemas educativos se han diversificado gracias a los continuos esfuerzos que se han realizado en materias de políticas públicas y educativas, sin embargo, existen en paralelo grupos sociales que se sienten estigmatizados y tienen la tendencia a percibir esto como una barrera para el proceso formativo en la educación superior ${ }^{8,9}$. Lo anterior vuelve necesario examinar el modo en que opera la idea de diversidad e inclusión en el proceso formativo de profesiones sanitarias. El presente artículo tiene como finalidad describir el fenómeno de la diversidad en el proceso formativo.

\section{Material y Método}

Este estudio cualitativo, aprobado por el Comité de Ética de la Universidad de Concepción, se basó en la teoría fundamentada ${ }^{10}$ perspectiva que realiza un análisis inductivo, donde los datos, el análisis y la teoría logran interrelacionarse con la finalidad de construir sus experiencias y el significado de ellas. Como técnica de recolección de datos se utilizó la entrevista semiestructurada y la entrevista grupal ${ }^{11}$. En el presente estudio participaron 11 docentes y 25 estudiantes de seis carreras de la salud: Medicina, Enfermería, Ki- nesiología, Fonoaudiología, Tecnología Médica y Obstetricia y Puericultura, pertenecientes a tres universidades chilenas de tres regiones diferentes. Los informantes clave fueron los jefes de las carreras estudiadas en las diferentes casas de estudio, previa autorización institucional y consentimiento informado individual.

Los criterios de inclusión para estudiantes fueron ser alumnos regulares de carreras de la salud que otorgaran el grado de licenciado y que hubiesen cursado al menos dos años en ella. Los criterios de exclusión correspondieron a casos ingresados a la carrera por transferencia de otra casa de estudios y con ausencias de clases por un período superior a tres meses consecutivos durante los últimos cuatro semestres académicos. Los criterios de inclusión para docentes fueron que impartieran clases sobre $21 \mathrm{~h}$ semanales y realizaran un curso semestral en una de las carreras de la salud seleccionadas, durante los últimos 5 años. El criterio de exclusión fue que su título no fuera sanitario, puesto que podrían carecer de la visión profesional y práctica de dicha disciplina.

Para el arranque muestral se identificó a alumnos por el criterio de máxima variación ${ }^{12}$. Para delimitar la muestra, se utilizó el muestreo teórico, cuyo criterio básico de selección es elegir a los casos que tienen mayor potencial de entregar información para el desarrollo de las categorías emergentes $^{13}$. Se incluyó participantes hasta alcanzar el punto de saturación teórica ${ }^{14}$. Para el análisis de datos se realizó codificación abierta, del método de comparación, identificando y describiendo inductivamente categorías ${ }^{10}$. Además, se resguardaron los criterios de rigurosidad científica propios del paradigma cualitativo: la credibilidad, transferibilidad y confirmabilidad ${ }^{15}$.

\section{Resultados}

Entre los resultados obtenidos emergió una categoría de análisis denominada "Formación en carreras de la salud en contextos de diversidad", que apunta a los aspectos actitudinales del proceso formativo y de las interacciones que se perciben en el proceso de enseñanza-aprendizaje en carreras de la salud. Un proceso que operaría en tres dimensiones necesarias: cognitiva, afectiva y conductual. Desde dichas dimensiones emergen 
dos subcategorías que permiten describir el fenómeno emergente: a) Diversidad de los actores del sistema y b) Modelo del profesional de la salud y docente ante la diversidad.

\section{Diversidad de los actores del sistema educativo}

Esta subcategoría describe, desde la percepción de los entrevistados, cómo son los actores del sistema de salud en el que se inserta la formación clínica. Primero emerge la idea de diversidad de pacientes en contextos clínicos. Los entrevistados señalan que se ven enfrentados a la atención de pacientes diversos, que pueden presentar vulnerabilidad social, situaciones de discapacidad física o cognitiva, ser inmigrantes, pertenecer a minorías sexuales o etnias; ante lo cual creen necesario cambiar las pautas de atención, para adaptarse al nuevo tipo de pacientes. Los participantes relatan que en atención primaria se puede ver más claramente la situación de vulnerabilidad, por medio de su apariencia personal y vestimenta, mientras que en el hospital, los pacientes socialmente vulnerables se pesquisan fácilmente si está indicado en su ficha (Tabla 1).

A su vez, los participantes identifican ciertos grupos en riesgo de poseer vulnerabilidad socioeconómica. Entre estos, están los inmigrantes, las personas provenientes de sectores rurales y las embarazadas privadas de libertad. Respecto a los primeros, comentan que a los inmigrantes que no están regularizados en su situación legal, se les califica como indigentes, permitiendo que tengan acceso a salud de igual manera.

En tanto, los pacientes provenientes de zonas rurales tienen otro tipo de riesgos a nivel de salud debido a la dificultad en el acceso a servicios de salud, especialmente aquellas pacientes embarazadas. Esto implica atenderles de manera distinta, por ejemplo, asegurando hospitalización, y que también se les enseñe a los estudiantes a integrar el aspecto biopsicosocial en sus atenciones con los pacientes.

Segundo, sobre el perfil del estudiantado y su experiencia en el contexto clínico, los alumnos reportan una serie de dificultades ligadas con la aparición de problemas de salud mental al llegar a quinto año, dificultades para poder respetar horarios de inicio de jornada de trabajo y para reconocer la jerarquía dentro de su lugar de trabajo. En tanto, los docentes comentan la presencia de problemas conductuales cuando los alumnos se encuentran realizando su internado, relacionados principalmente con el respeto de las normas de cada centro de práctica. En relación con las expectativas acerca de la realidad profesional, los entrevistados mencionan que los estudiantes al realizar sus prácticas clínicas se van desilusionando al darse cuenta de que la realidad profesional es distinta a como se les había enseñando y, junto con esto, se van contaminando con las actitudes de funcionarios que ocasionalmente no respetan los protocolos de atención. En el internado se puede diferenciar cuáles estudiantes llegan motivados y se esfuerzan por atender. Los docentes entrevistados valoran la proactividad de algunos estudiantes al momento de realizar sus prácticas clínicas, lo que, junto con la presencia de liderazgo innato, facilita la vinculación con pacientes.

Tercero, sobre la caracterización de los profesionales de la salud, los entrevistados comentan que la vocación en estos va menguando y se va viendo afectada por intereses de ingresos económicos y el desarrollo del prestigio profesional. Esta pérdida de la vocación va dada por la influencia de otros profesionales de la salud en las prácticas clínicas, o luego de haber egresado, lo que opera como un eje principal en la experiencia formativa que tendrán dichos profesionales en el contexto clínico. Otros actores, a su vez, relacionan aspectos tales como la religión o aspectos de personalidad, que serían clave para definir la vocación de servicio en un profesional.

\section{El modelo del profesional de la salud y docente ante la diversidad}

La vinculación entre los distintos actores del sistema de salud público puede comprenderse mejor abordando el rol de modelo que evidencian los estudiantes en los profesionales de la salud y en sus docentes. En opinión de algunos docentes, en ocasiones existe disposición negativa de algunos miembros del equipo de salud a atender a cierto tipo de pacientes cuando se ven sobrepasados por el tipo de atención que requieren, lo que demanda sensibilizar a dichos equipos (Tabla 2).

Con respecto a lo anterior, aparecería una disposición negativa al atender a pacientes en caso de presentar condiciones genéticas complejas, ocasiones en que se ha podido presenciar que algunos profesionales eluden la atención. Los participantes atribuyen esta actitud a la exigencia de 
Tabla 1. Citas asociadas al análisis de la codificación abierta. Subcategorías: Diversidad de los actores del sistema educativo

\section{Vulnerabilidad de Pacientes:}

"En atención primaria, yo creo que uno puede... ver más vulnerabilidad o darse cuenta más, porque claro, uno ahí ve... como viene la persona... su aspecto icierto? Como viene vestida, ahí uno puede inferir, muchas veces, situaciones de vulnerabilidad" [Entrevistado 8, docente]

"(...) Sí, personas que son deprivadas desde el punto de vista educativo, o sea la mayoría no ha terminado el colegio, además viven situaciones precarias graves. Graves, o sea, pacientes que comían tres papas al día, situaciones económicas complejas, eh y de acceso a la información. La gente no sabía mucho cómo funcionaba el sistema de salud, cuáles eran sus derechos ite fijai? Que también se unía un poco a lo otro. Ese tipo de personas veíamos. (...) Lo que pasa es que el ochenta por ciento de las personas eran asi" [Entrevistado 3, docente]

"Mira yo creo que una de las cosas qué a mí no me gusta, que siento que son más desfavorecidos, siento que es donde se vulneran más derechos. Es principalmente a mujeres qué están presas (...) da pena verlas cuando están en el hospital que, por el protocolo tienen qué estar los gendarmes afuera de... de la sala, pero las dejan con las esposas a las camas, y eso de repente resulta un poco chocante" [Entrevistado 11, docente]

"(...) Si es rural, lo más probable es que la dejemos hospitalizada al tiro. A pesar de que puede estar iniciando recién. ¿Cierto? Porque pensamos que si se va, va a tener parto en la casa, o va a tener problemas, no va a poder volver, a medianoche, no tiene la locomoción, entonces hay que estar viendo esas variables también para, tomar una decisión, eh apropiada (...) porque tiene otras circunstancias de acceso, de, de que no tiene plata para trasladarse, de que no tiene el apoyo a lo mejor (...), entonces hay muchas otras situaciones que hay que tomar en cuenta para decidir, la conducta a seguir" [Entrevistado 8, docente]

\section{Experiencia de los estudiantes:}

"A ellos les cuesta mucho entender que el horario de inicio de la jornada es a las ocho de la mañana, significa que a los ocho hay que estar listos para empezar a trabajar, y no llegar corriendo, diez minutos después; es que me voy a acomodar, me voy a cambiar ropa, es que voy a tomar desayuno. Esas cosas, les cuesta muchísimo, y el tema del trato, de la jerarquía, no la reconocen" [Entrevistado 4, docente]

\section{Experiencia de los estudiantes:}

"Bien, doctores que son docentes que son monitores y que, llegan atrasados, o tienen una actitud funcionaria para cumplir, (...) se empiezan a desilusionar, bueno por supuesto que no con todo el mundo, pero empiezan a ver que la realidad no es idílica como se les había venido enseñando en la escuela, que la vocación antes que nada y que la realidad es algo distinta. Médicos funcionarios que cumplen con los horarios, para cumplir con el número de pacientes, pero que no tiene la dedicación al paciente que ellos esperaban; y ahí empiezan ellos a contaminarse" [Entrevistado 2, docente]

"La motivación. O sea, hay estudiantes que llegan al área, porque es por sorteo generalmente la asignación de los internados. Entonces se nota mucho quienes están motivados, que se esfuerzan por atender, estudian, preguntan, se las ingenian" [Entrevistado 7, docente]

"Lo vemos que, por ejemplo, nosotros tenemos que ir de primero a los, CESFAM, eh, y en los informes en vez, además de escribir la diferentes cosas que hacemos, nos hacen dar nuestra opinión de las cosas que se pueden mejorar, de las cosas que ya tienen buenas, entonces, como la retroalimentación es distinta" [Entrevista grupal 1, participante 7]

\section{Profesional de la salud:}

"(...) creo que fue en Navarra, se le hizo una encuesta, (...) una encuesta de por qué quisiste ser médico y ponían una serie de opciones, (...). Cómo va cambiando el asunto, desde que los médicos quieren curar a las personas y al segundo año de clínica se dan cuenta que no se puede curar a las personas o sea se curan algunas, otras se mantienen no más, entonces ya te empiezas a desilusionar (...) desgraciadamente se ha ido más hacia la parte económica y hacer prestigio" [Entrevistado 1, docente]

"(...) la gran mayoría de los alumnos ingresan con una vocación de servicio y la van perdiendo más adelante, debido fundamentalmente, a lo que observan, o sea cuando ya empiezan a ir a campos clínicos y se empiezan a decepcionar muchas veces de las conductas de los profesionales de la salud, que no es coherente con lo que se les enseñaba en las clases" [Entrevistado 2, docente] 
Tabla 2. Citas asociadas al análisis de la codificación abierta. Subcategorías: El modelo del profesional de la salud y docente ante la diversidad

\section{Discapacidad:}

"Pero el equipo se veía bien sobrepasado. La sensación que tenían y que a mí me transmitían era que tenían tantas cosas que hacer, era tan desgastante el trabajo con todos los pacientes, que además ponerle más atención especial a un paciente en particular los desgastaba tremendamente. Y era como "chuta, más encima tengo que explicarle a este mil veces" [Entrevistado 3, docente]

"En general... cuando hay problemas muy complejos, a mí me tocó en pediatría de pronto... chicos que tenían trastornos genéticos muuuy graves, muy complejos... Eh... que lo que veía yo, que en general los pediatras se corrían de atenderlo" [Entrevistado 5, docente]

"es que era la enfermera, yo te diría que eran bastante... +++ bueno ellas son imuy maternales! iCon todo el mundo po! (sic) Jaja, así que eran mucho más maternales con estos chicos" [Entrevistado 5, docente]

"Entonces era muy difícil poder comunicarse con estas personas entonces el equipo por lo menos, la queja que tenían era que tenían que explicarle una, dos, tres, cuatro, cinco veces lo mismo" [Entrevistado 3, docente]

"¿sabes qué, necesito otra muestra?; oye, es que el paciente tiene esta condición, que cuesta tanto. Ya, entonces, con lo que tengo voy a hacer lo que pueda, y voy a informar ¿qué es lo que más te interesa?; ¿Qué es lo que privilegio?" [Entrevistado 4, docente]

"Si me llega un paciente sordomudo... yo puedo tener dos actitudes: ah, yo no lo puedo atender, que lo vea otro.. o, yo hacer todo esto que estamos conversando, llamar a la mamá... llamar a... alguien se tiene que comunicar en el mundo con él, porque si no estaría muerto de hambre... bueno buscar a esa persona, para poder comunicarme con él, pero eso significa un esfuerzo extra, porque no está dentro de la estructura que tiene nuestro sistema de salud para trabajar" [Entrevistado 9, docente]

\section{Inmigrantes:}

"Por ejemplo los pediatras no se toman la molestia de saber cómo es la alimentación en Haití, y le indican las pautas de alimentación a una guagua... chilenas. Alimentos que no han comido en su vida y cómo se la van a dar" [Entrevistado 5, docente]

"y el principal problema que tuve, porque primero, desconocía absolutamente su cultura, era africano; no me podía comunicar con él, no tenía idea de lo que le pasaba por la cabeza, y en algún minuto él se desespera, y lo que él me exigía era que yo le devolviera su apéndice... porque él no podía perder algo de su cuerpo... " [Entrevistado 9, docente]

\section{Orientación sexual:}

"(...) A mí me ha tocado muchas veces que los docentes asumen la orientación sexual de los pacientes y después yo hablando con los pacientes me doy cuenta que no era efectivamente lo que ellos creían y tengo que reorientar toda la consejería después. Obviamente es un caos porque eso significa que estamos fallando como proveedores de la salud después a futuro. Y eso es lo que se nos está enseñando y esa yo creo que es la falla, que no existe una instrucción de parte de los docentes respecto del tema y es porque yo creo que nunca se vio la necesidad. Que durante mucho tiempo se quiso tapar el sol con un dedo" [Entrevista grupal 2, participante 2]

"el gran tema que tenemos con las personas... viviendo con VIH, que... tenían una extrema discriminación... tremenda, tremenda. Y a medida que fuimos estudiando vimos que no era tanto por el temor al VIH, sino que porque, la gran mayoría, eran homosexuales" [Entrevistado 5, docente]

"este señor tiene VIH, generalmente nosotros con los alumnos no lo vamos a atender porque si es un parto va, va a ser por vía cesárea, no por parto vaginal, y eh... generalmente la atención va más a las precauciones que hay que tener. Pero no, no se trata de manera más especial" [Entrevistado 11, docente]

\section{Religión:}

"Algunos como que... rechazan de plano, o sea que no puede ser, no puede ser que, que alguien vaya a morir por sus creencias religiosas, algunos rechazan de plano. Y otros, empiezan... ya, por la... por convencer a la persona, a la familia... darle fundamentos, ¿cierto? Un poquito negociar esa parte hasta que la persona trata de ver el otro lado. Principalmente hablar con la familia, claro, por supuesto que, si la familia se niega, no hay caso. ¿Cierto? pero, se trata siempre de convencer a la familia... de... de ver qué, que la vida es más importante" [Entrevistado 8, docente]

"Solo recuerdo alguna oportunidad, alguna alumna que tuvimos hospitalizada, que tenía esa creencia, y estaba como bien complicada la chica, no me acuerdo que tenía, pero, estuvo bien complicada, y al final, eh... no sé si conversó el equipo de salud con la familia o no, pero al final se transfundió" [Entrevistado 8, docente]

"Es un tema, la religión, que me pasa por el lado. No me pasa por el lado, o sea, sí me pasa, sí me pasan cosas por... de nuevo el poder, el poder que tiene la religión en este país, que influye en las personas, influye en los profesionales, en las prácticas finalmente, ahí sí que me toca" [Entrevistado 5, docente]

"bueno, lo que tendría que contestarle es lo que... que es el diálogo que teníamos en el tema del VIH muchas veces con personas que eran extremadamente religiosas o de líneas muy duras. Que una cosa es tú creencia para tu vida personal, y otra cosa es lo que tú tienes que hacer en tu rol profesional... una cosa no tiene que ver con la otra. Por qué, porque en tu rol profesional tú tienes que darle una respuesta a la persona, que te está pidiendo una ayuda o que necesita una atención. Y esa respuesta no puede estar mediada por tu creencia religiosa" [Entrevistado 5, docente] 
tener que hablar con los padres del paciente para explicar un mal pronóstico y la forma de eludir la atención se manifiesta derivando al paciente a otro profesional. Empero, también se presentan situaciones similares con profesionales del equipo de salud, en la atención a pacientes pediátricos con discapacidad intelectual o en situaciones particulares de personas con discapacidad, en las que se evidencia una preocupación especial en la atención a este tipo de pacientes, para lograr una atención de calidad. Relatan que para superar las dificultades que se encuentran en la atención de pacientes con déficit cognitivo, con los cuales la comunicación es difícil, la solución puede ser crear estrategias para mejorar la comunicación y contener afectivamente al paciente.

Para estos casos mencionan que también es necesario respetar los protocolos de los laboratorios clínicos y, por ejemplo, cuando es necesario repetir las muestras debido a las dificultades con los pacientes, se maneja su situación sin hacer discriminación. Algunos docentes entrevistados relatan que una forma de relacionarse con pacientes que presentan dificultades comunicativas es utilizar el humor, para distender el ambiente y disminuir la angustia del paciente. En ocasiones, también se presenta la dificultad de comunicación con pacientes sordos, en cuyo caso, el profesional debe hacer un esfuerzo extra para atenderlo, derivándolo o recurriendo a una tercera persona que posibilite la comunicación, dado que este tipo de atención no está considerada en la estructura del sistema de salud.

Otro tipo de pacientes en particular son los inmigrantes, donde ocasionalmente se percibe falta de empatía desde los profesionales, por ejemplo, en el caso de prescribir cierto tipo de alimentación que ellos desconocen, presentándose también dificultades en la comunicación cuando existen barreras idiomáticas, lo que muchas veces se resuelve utilizando el traductor del teléfono móvil o algún familiar que realice la traducción. Pero, en el caso de algunos pacientes extranjeros, la dificultad de atención va más allá de una barrera idiomática, presentándose diferencias culturales importantes que hacen difícil una adecuada atención, como ciertas creencias respecto a las cirugías. Indican que esto se atribuye a que la estructura administrativa de las instituciones de salud no está preparada para incluir culturas diferentes a la dominante.

Las barreras culturales también se dan en la atención de pacientes provenientes de otras etnias o de zonas rurales. Esto, además de la dificultad de comunicación, implica que deban derivarse horas para atenderse en hospital. Lo anterior causa que las atenciones se vean frustradas porque el profesional tratante no entiende qué le aqueja a su paciente, y las derivaciones no siempre están disponibles para entender las hipótesis diagnósticas, quedando evidenciada una falla del sistema de salud. Así, pese a la existencia de facilitadores interculturales, el proceso de atención se ve dificultado por la comunicación, las creencias de cada etnia y la vulnerabilidad económica y social de estos pacientes.

Adicionalmente, en general se percibe discriminación por la orientación sexual de los pacientes. En ocasiones, la orientación se asume por parte de los profesionales sanitarios. Esto resulta dificultoso al momento de otorgar consejerías, debido a que no siempre aquella suposición se condice con la verdadera opción sexual. En opinión de los entrevistados, también existe disposición negativa ante pacientes portadores de VIH, lo cual ocurre no por temor al VIH, sino cuando son homosexuales. Sumado a esto, se suele evitar involucrar a los estudiantes en la atención de pacientes con este tipo de diagnóstico, por las precauciones de salud o riesgo de contagio que son necesarias tener.

Respecto a aquellos pacientes religiosos, los entrevistados plantean que el valor de la vida es mucho más importante que las creencias, por lo que intentan convencer a la persona de recibir un determinado tratamiento. Por último, algunos participantes reconocen que la religión influye en el ejercicio profesional de manera negativa, sin embargo, se reconoce que las respuestas profesionales a los pacientes no pueden estar influenciadas por la propia religión, debiendo ser capaces de separar la vida personal de la profesional para aceptar la diversidad.

\section{Discusión}

A partir de los resultados obtenidos es posible reconocer algunos aspectos distintivos del tipo de diversidad que opera actualmente en el proceso formativo en carreras de la salud. Esto hace necesario considerar que el aprendizaje clínico en salud no debe restringirse únicamente a los aspectos técnicos de la formación profesional, sino que, a su vez, debe considerar una formación 
social que permita a los estudiantes reconocer las diferencias tanto de necesidades de salud como las socioculturales de los pacientes. En un escenario tal, la formación clínica debiese propender a reconocer al otro en torno a la diversidad: una educación inclusiva tiene por principio el formar graduados que se orienten a la comunidad y sean socialmente responsables ${ }^{16}$.

Escenario complejo, pues desde la academia, la diversidad se ha desarrollado principalmente desde la dimensión de discapacidad, donde existen más estudios y se han definido paradigmas conceptuales. Así, históricamente se han desarrollado diversos modelos de trato social a personas con diversidad funcional, que van desde el modelo de prescindencia, que considera la discapacidad como castigo divino y propone acabar o marginar a estas personas, el rehabilitador, que consideraba la discapacidad como una imperfección que hace imprescindible rehabilitar al individuo, hasta el modelo social, que considera la discapacidad como una anormalidad de la sociedad y no del sujeto ${ }^{17,18}$.

Este último modelo es el que se considera en las aproximaciones más recientes hacia otras formas de diversidad, y debería promoverse en la formación universitaria. No obstante, esto no solo requiere un cambio actitudinal, sino el aprendizaje de estrategias para incluir al otro cuando, por ejemplo, su comunicación, su etnia o su credo son distintos a los propios.

Dicho de otro modo, la inclusión educativa es una cuestión importante a tener en cuenta por las universidades, particularmente en el proceso formativo clínico, donde es posible observar distintas dimensiones de diversidad asociadas a la vulnerabilidad social, la inmigración, la discapacidad, la orientación sexual y la religión del usuario ${ }^{19}$, como también la diversidad de experiencias y trayectorias de profesionales y alumnos.

\section{Referencias}

1. Illesca M, Lagos X, Navarro N. Modelos de formación docente base para una innovación curricular. Rev Educ Cienc Salud 2005; 2 (2): 93-6.

2. Fasce E. ¿ Son suficientes las competencias profesionales en la enseñanza de las ciencias de la salud? Rev Educ Cienc Salud 2008; 5 (2): 81.

3. Schonhaut L. Educación multiprofesional como estrategia para la atención primaria de salud: aprendiendo juntos para trabajar en equipo. Rev Educ Cienc Salud 2007; 4 (1): 29-31.

4. Valdés de la Rosa C, Iglesias H, Durán M, Gayol A, Hernández M. Estrategia curricular para la formación pedagógica en la carrera de Medicina. Rev Hum Med 2010; 10 (2): 0-0.

5. Matus O, Ortega J, Parra P, Ortiz L, Márquez C, Stotz $\mathrm{M}$, et al. Condiciones del contexto educativo para ejercer el rol docente en Ciencias de la Salud. Un enfoque cualitativo. Rev Med Chile 2017; 145 (7): 926-33.

6. Ortega-Bastidas J, Matus-Betancourt O, Parra-Ponce P, Oyarzo-Pereira J, Oliva-Montero C, Henríquez-Valenzuela $\mathrm{Y}$, et al. Roles y habilidades docentes según escenarios educativos en carreras de la salud. Educación Médica Superior 2018; 32 (4): 121-30.

7. Visser J, Stokes S. Is education ready for the inclusion of pupils with emotional and behavioural difficulties: A rights perspective? Educ Rev 2003; 55 (1): 65-75.

8. Stevenson J. Internationalisation and religious inclusion in United Kingdom Higher Education. High Educ Q 2014; 68 (1): 46-64.

9. Tupan-Wenno M, Camilleri AF, Frölich M, King S. Effective Approaches to Enhancing the Social Dimension of Higher Education. Knowledge Innovation Centre (Malta) 2016. Disponible en: https://www. pedocs.de/volltexte/2016/12400/pdf/Ideas_2016_Effective_Approaches_to_Enhancing_the_Social_Dimension_of_Higher_Education.pdf. [Consultado el 24 de abril de 2019].

10. Strauss AL, Corbin J, Zimmerman E. Bases de la investigación cualitativa. Técnicas y procedimientos para desarrollar la teoría fundamentada. Medellín, Colombia: Editorial Universidad de Antioquia; 2002.

11. Vieytes R. Metodología de la investigación en organizaciones, mercado y sociedad. Buenos Aires, Argentina: Editorial de las Ciencias; 2004.

12. Patton MQ. Qualitative evaluation methods. Beverly Hills, USA: Sage Publications; 1980.

13. Rodríguez G, Gil J, García E. Metodología de la investigación cualitativa. Málaga, España: Alibe; 1999.

14. Glaser B, Strauss A. The Discovery of Grounded Theory: Strategies for qualitative research. New York, USA: Aldine; 1967.

15. Lincoln Y, Guba E. Naturalistic inquiry. Thousand Oaks, Ca, USA: Sage; 1985.

16. Nunan T, George R, McCausland H. Inclusive education in universities: why it is important and how it might be achieved. Int J Incl Educ 2000; 4 (1): 63-88.

17. Palacios A, Romañach J. El modelo de la diversidad: la bioética y los derechos humanos como herramientas para alcanzar la plena dignidad en la diversidad funcional. 
Santiago de Compostela, España: Ediciones Diversitas; 2006.

18. Palacios A, Romañach J. El modelo de la diversidad: una nueva visión de la bioética desde la perspectiva de las personas con diversidad funcional (discapacidad). Intersticios. Revista sociológica de pensamiento crítico 2008; 2 (2): 37-47.
19. Matus-Betancourt O, Schilling-Norman MJ, Ortega-Bastidas J, Pérez-Villalobos C, McColl-Calvo P, Espinoza-Parcet $\mathrm{C}$. Higher education inclusion and its dimensions: A theoretical proposal. MedEdPublish 2018; 7 (1): 0-0. Disponible en: https://doi.org/10.15694/ mep.2018.0000029.1. [Consultado el 24 de abril de 2019]. 